\title{
Estratégia\&Negócios
}

ISSN 1984-3372

http://www.portaldeperiodicos.unisul.br/index.php/EeN/

\section{MAPEAMENTO DE ABORDAGENS EPISTEMOLÓGICAS EM PUBLICAÇÕES SOBRE ESTRATÉGIA ORGANIZACIONAL}

\section{MAPPING OF EPISTEMOLOGICAL APPROACHES ON PUBLICATIONS ON THE ORGANIZATIONAL STRATEGY FIELD}

\section{Renê Birochi}

Professor de Pós-Graduação em Administração pela Universidade do Sul de Santa Catarina - UNISUL.

E-mail: rene.birochi@unisul.br

\section{Maria Zenilda da Silva}

Mestranda em administração pela Universidade do Sul de Santa Catarina - UNISUL.

E-mail: mariazenildasilva@hotmail.com

\section{Flavia Domingues}

Mestranda em administração pela Universidade do Sul de Santa Catarina - UNISUL.

E-mail: flaviadomingues10@gmail.com

\section{Amilton Bento}

Mestrando em administração pela Universidade do Sul de Santa Catarina - UNISUL.

E-mail: vendas@mpci.com.br

\section{Richeli Sacheti}

Mestranda em administração pela Universidade do Sul de Santa Catarina - UNISUL.

E-mail: coach@richelisachetti.com.br

Recebido em 03/12/2012. Aprovado em 23/02/2013. Disponibilizado em 04/03/2013. Avaliado pelo Sistema double blind review

R. eletr. estrat. neg., Florianópolis, v.5, n.3, p. 259-275, set./dez. 2012 http://portaldeperiodicos.unisul.br/index.php/EeN/index
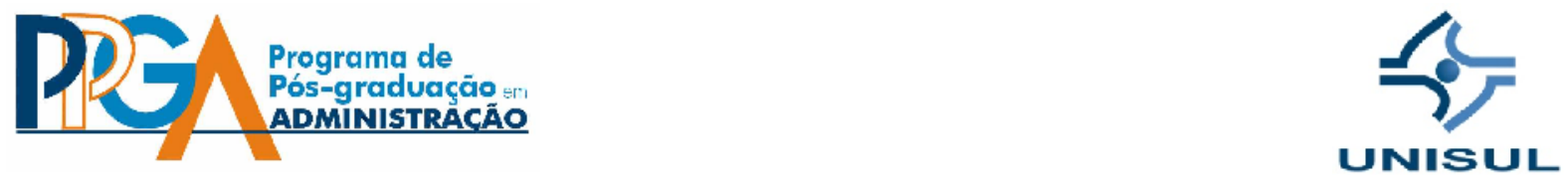

CCopyright 2008 UNISUL-PPGA/Estratégia e Negócios. Todos os direitos reservados. Permitida citação parcial, desde que identificada a fonte. Proibida a reprodução total. Em caso de dúvidas, consulte o editor: ademar.unisul@gmail.com; (48) 3229-19 


\section{RESUMO}

Este artigo tem como objetivo realizar um estudo sobre as principais abordagens epistemológicas, identificadas em artigos sobre o tema da estratégia organizacional. 0 referencial teórico que guiou esta investigação é baseado na clássica definição proposta por Burrell e Morgan (1979) a respeito dos quatro paradigmas de pesquisa em ciências sociais. Foram investigados artigos da área de Administração, Ciências Contábeis e Turismo, publicados em periódicos nacionais entre os anos de 2001 a 2012, nos estratos B1 e B2 do sistema Qualis/Capes. Visando a atender este objetivo, foi realizado um estudo bibliométrico para mapear as publicações selecionadas. Como resultado deste estudo, constatou-se a predominância do paradigma funcionalista, assim como uma reduzida presença de artigos que explicitam as suas escolhas teórico-metodológicas, como fundamento para seus estudos empíricos.

Palavras-chaves: Estratégia Organizacional. Paradigmas. Teoria das Organizações. Epistemologia.

\section{INTRODUÇÃO}

A predominância da epistemologia positivista nas pesquisas na área de administração é marcante. Segundo Cruz e Pedrozo (2008), tais pesquisas fundamentam-se em pressuposições tais como a da neutralidade do pesquisador em relação ao seu objeto de estudo, garantida pela utilização de técnicas e procedimentos metodológicos que asseguram a objetividade de suas análises. Perret e Séville (2003) acrescentam que o esforço está em enclausurar o objeto para, dessa maneira, entender a realidade estudada. As motivações subjacentes a estes procedimentos vinculam-se com a pretensa busca pela objetividade do conhecimento científico, cujo objetivo central visa a encontrar relações causais e regularidades entre os fenômenos estudados.

Por outro lado, observa-se a presença de novas epistemologias entre os pesquisadores da área de administração, apesar, ainda, de sua baixa representatividade. Para Perret e Seville (2003), é possível notar a emergência em trabalhos de cunho R. eletr. estrat. neg., Florianópolis, v.5, n.3, p. 259-279, set./dez. 2012 
construtivista, cuja origem remonta a estudos etnográficos de antropólogos norteamericanos no início do século XX. De acordo com Thiollent e Bourdieu (1996) esta é uma postura alternativa ao positivismo, que visa a compreender como o sujeito do conhecimento interpreta a realidade e a modifica na sua interação com o objeto empírico, possibilitando a constituição de novas categorias de significado. O construtivismo, segundo esses autores, valoriza a imersão do investigador no fenômeno estudado, encorajando a relação dialógica entre o pesquisador e o objeto.

O debate a respeito das diferenças epistemológicas na pesquisa em ciências sociais remonta ao clássico artigo de Burrel e Morgan (1979), que influenciou largamente as pesquisas em administração, a partir dos anos 1980 (CALDAS; BARTERO, 2007). Burrel e Morgan (1979) propuseram um modelo para agrupar as fundações teóricas das pesquisas, a partir de características e escolhas teórico-metodológicas. O modelo agrupa as teorias em quatro paradigmas principais - humanismo radical, estruturalismo radical, interpretativo e funcionalismo - constituídos a partir de dois eixos ortogonais: a dimensão objetiva-subjetiva, e a sociologia da regulação e da mudança radical. No entanto, independentemente do paradigma adotado pelo pesquisador, as suas pesquisas devem procurar explicitar quais são os pressupostos teórico-metodológicos que fundamentam as suas investigações (CALDAS; BARTERO, 2007).

Sendo assim, este artigo pretende contribuir para a reflexão sobre os fundamentos epistemológicos que constituem os estudos sobre estratégia organizacional. Neste contexto, a indagação de pesquisa que orientou as análises aqui realizadas é expressa pelas perguntas: Quais são os paradigmas mais utilizados nos estudos sobre o tema da estratégia organizacional? E quais são as palavras-chave empregadas nessas pesquisas, que evidenciam os pressupostos teórico-metodológicos adotados?

Assim, para que se possa responder a essas duas perguntas, este artigo tem como objetivo analisar os paradigmas dominantes nos estudos sobre estratégia organizacional e apontar as terminologias mais frequentes nos artigos que contribuem para a identificação paradigmática, utilizando a análise bibliométrica e delimitando os periódicos dos estratos B1 e B2 no período abrangido entre 2001 e 2012. O objetivo principal foi decomposto nos seguintes objetivos específicos: (i) identificar os principais paradigmas de R. eletr. estrat. neg., Florianópolis, v.5, n.3, p. 259-279, set./dez. 2012 
pesquisa em artigos publicados nos periódicos classificados nos estratos B1 e B2 do Sistema Qualis/Capes; (ii) identificar os autores mais citados entre os paradigmas dominantes; (iii) identificar os principais temas abordados nesses periódicos; e, (iv) identificar as principais teorias que fundamentam os artigos estudados.

A relevância deste estudo justifica-se na medida em que este artigo pretende contribuir para o debate sobre os fundamentos teórico-metodológicos que fundamentam os estudos em estratégia organizacional. Para tanto, o artigo está estruturado nas seguintes seções: (i) Introdução; (ii) Referencial Teórico; (iii) Procedimentos Metodológicos; (iv) Análise dos Resultados; e, (v) Considerações Finais.

\section{REFERENCIAL TEÓRICO}

Para fundamentar a sua proposta teórica, Burrel e Morgan (1979) realizaram uma revisão do conceito de paradigma, popularizado por Kuhn (1962). Segundo os autores, este conceito foi exposto a uma ampla e confusa variedade de interpretações, devido, em parte, ao próprio Kuhn, que propôs o conceito em, pelo menos, 21 modos distintos (MASTERMAN, 1970), e consistente com três sentidos amplos do termo: (1) como um modo de ver, ou visão cabal da realidade; (2) como referência à organização social da ciência, em termos de escolas de pensamento associadas a tipos particulares de realizações científicas; e (3) como atividades de resolução de quebra-cabeças científicos (CALDAS; BERTERO, 2007).

A abordagem a respeito da distinção entre as diferentes correntes teóricas nos estudos sobre as organizações tem como premissa a visão de que todas as teorias organizacionais estão baseadas, por um lado, em uma filosofia da ciência e, por outro, em uma teoria da sociedade (BURREL; MORGAN, 1979). Os autores descrevem ainda que, embora os teóricos organizacionais nem sempre tenham clareza sobre os desígnios básicos que fundamentam os seus pontos de vista, todos adotam uma posição baseada na filosofia da ciência e na teoria da sociedade, que suportam teoricamente as suas abordagens. Estejam ou não conscientes disto, trazem para seus temas de estudos um quadro de referência que reflete uma série de pressupostos sobre a natureza do mundo social e da maneira como este deveria ser investigado. 
Segundo Burrel e Morgan (1979), as pesquisas em ciências sociais são constituídas em função de quatro conjuntos de pressupostos referentes à ontologia, à epistemologia, à natureza humana e à metodologia. Os cientistas sociais abordam suas disciplinas por meio de pressupostos explícitos ou implícitos sobre a natureza do mundo social e a maneira como este pode ser investigado. Os pressupostos ontológicos referem-se à natureza da realidade investigada. Ou seja, os cientistas sociais definem a questão ontológica tendo em vista explicitar os pressupostos que constituem a "realidade", seja ela considerada como algo externo ao pesquisador - impondo-se à consciência "de fora" para "dentro" - ou, por outro lado, como um produto da própria consciência do investigador; se a "realidade" tem uma natureza objetiva ou se é produto de cognição individual; se a "realidade" é um dado externo ou se é um produto da mente humana.

O segundo conjunto de pressupostos refere-se aos fundamentos epistemológicos da ciência, que são definidos através da explicitação da natureza mesma que constitui o conhecimento científico. Os pressupostos epistemológicos são separados em dois eixos principais: uma dimensão objetiva do conhecimento científico e uma dimensão subjetiva (BURREL; MORGAN, 1979). A perspectiva objetiva do conhecimento vincula-se aos modelos dominantes de produção do conhecimento, que postulam que o conhecimento simplesmente reflete o mundo tal qual ele é (CHAUÍ, 2000). Por outro lado, a perspectiva epistemológica subjetiva (ou subjetivista), tem origem na fenomenologia de Edmund Husserl, na qual o conhecimento está relacionado aos atos interpretativos do sujeito (PRASAD, 2005), que fazem com que o pesquisador imprima significados aos objetos de seu estudo. O processo de significação e de intencionalidade dos atos do sujeito do conhecimento está, assim, intimamente vinculado ao processo de subjetivação da realidade. Ou seja, a realidade do conhecimento não é considerada independente do sujeito, mas, ao contrário, faz parte inerente do processo de significação empreendido pelo sujeito na sua interação com o objeto do conhecimento.

Como terceiro conjunto de pressupostos relativos à natureza humana, Burrel e Morgan (1979) definem como sendo um conjunto de relações constitutivas entre o homem e o ambiente, tendo em vista a primazia de um sobre o outro (e vice-versa). São perspectivas em ciências sociais embasadas em uma visão humana que resulta em uma ação determinista 
às situações encontradas no mundo externo. Nessa perspectiva, os pesquisadores e suas experiências são produtos do ambiente, condicionados pelas circunstâncias externas, onde as forças externas, autônomas e formais moldam as mudanças organizacionais. Por outro lado, a perspectiva voluntarista considera que as mudanças organizacionais são o resultado de decisões individuais (da agência humana).

Os diferentes pressupostos sobre a ontologia, a epistemologia ou a natureza humana da ciência, conduzem, também, a metodologias diferentes entre si. Podem-se identificar, assim, metodologias nas ciências sociais que tratam dos fenômenos sociais como se fossem fenômenos do mundo da natureza: como dados reais, externos; e outras metodologias que atribuem a estes fenômenos atributos pessoais e subjetivos.

Os pressupostos sobre a natureza das ciências sociais podem ser representados em quatro quadrantes constituídos pelas dimensões subjetivo/objetivo e regulação/mudança radical. As duas dimensões resultam, assim, em quatro paradigmas sociológicos: humanismo radical, estruturalismo radical, interpretativo e funcionalismo (Figura 1).

Figura1 - Paradigmas na Análise Organizacional

MUDANÇA RADICAL

\begin{tabular}{|c|c|c|}
\cline { 2 - 3 } SUBJETIVO & $\begin{array}{c}\text { HUMANISMO } \\
\text { RADICAL }\end{array}$ & $\begin{array}{c}\text { ESTRUTURALISMO } \\
\text { RADICAL }\end{array}$ \\
\cline { 2 - 3 } & INTERPRETATIVO & FUNCIONALISMO \\
\hline
\end{tabular}

OBJETIVO

Fonte: Adaptado de Burrell e Morgan (1979, p. 22).

\section{REGULAÇÃO}

Ao identificar quatro paradigmas na teoria social, os autores sugerem, essencialmente, que é importante analisar a produção de uma determinada área do conhecimento em função dos quatro conjuntos básicos de pressupostos teórico- 
metodológicos. Cada um deles, assim, define a extensão de um determinado "território intelectual".

O paradigma funcionalista busca a explicação racional da atividade social. Composto de artefatos empíricos concretos cujas inter-relações podem ser identificadas, estudadas e medidas através de instrumentais derivados das ciências naturais.

No âmbito do paradigma funcionalista, os teóricos das organizações abordam frequentemente seu objeto a partir de uma estrutura de referências com base em pressupostos não discutidos, e, na medida em que esses pressupostos são reforçados e reafirmados continuamente por diversos pesquisadores, essa visão ortodoxa de mundo passa a ser assumida como única e inquestionável (SERVA; DIAS; ALPERSTEDT, 2010, p. 1).

O paradigma interpretativo concebe o mundo social como um processo que é fruto da atividade dos indivíduos. Os problemas relativos ao conflito, dominação, contradição, potencialidade e mudança não encontram lugar em seus quadros de referência. Eles estão orientados para a compreensão de um mundo social subjetivamente criado, tal como este se apresenta em termos de um processo continuado. A sociologia interpretativa centra-se na compreensão da essência da vida cotidiana. Cruz e Pedrozo (2008, p. 60) afirmam que:

Sujeito e objeto são interdependentes. Sendo assim, o conhecimento gerado é sempre subjetivo e particular a um contexto. Para os interpretativistas, o processo de criação do conhecimento passa pela compreensão do senso que os atores envolvidos dão à realidade. Quem adota essa postura busca compreender a realidade por meio das interpretações feitas pelos atores. Assim, os interpretativistas diferenciam compreensão de explicação, ao contrário dos positivistas que consideram ambas de forma igual.

O paradigma humanista radical pretende superar as noções fundadas na falsa consciência (a alienação do sujeito do conhecimento), que inibe ou impede a verdadeira consciência do homem em relação ao mundo que o circunda (a consciência histórica de si). O eixo central desta abordagem está baseado na ruptura cognitiva do indivíduo em relação às estruturas sociais, que alienam o sujeito do conhecimento (BURREL; MORGAN, 1979).

Por fim, o paradigma estruturalista radical fundamenta-se em abordagens baseadas na mudança radical, a partir de uma perspectiva objetivista. Se, por um lado, os R. eletr. estrat. neg., Florianópolis, v.5, n.3, p. 259-279, set./dez. 2012 
humanistas radicais constroem suas abordagens baseadas na consciência do sujeito do conhecimento (o voluntarismo da agência humana), visando a endereçar uma crítica radical à sociedade, por outro lado, os estruturalistas radicais concentram-se em superar as relações estruturais preexistentes (o determinismo das estruturas sociais), fundadas em abordagens realistas. Nesse sentido, enfatizam que a mudança radical faz parte da própria natureza e estrutura da sociedade contemporânea, e buscam explicar as inter-relações que subjazem no contexto das estruturas sociais.

Diversas são as formas utilizadas para classificar as posturas epistemológicas de pesquisa na área de administração e o estudo dos principais autores aqui citados oportunizam novas reflexões à consolidação ao esforço de pesquisa aqui realizado. Na seção seguinte apresentaremos os procedimentos metodológicos adotados na pesquisa realizada.

\section{PROCEDIMENTOS METODOLÓGICOS}

Este artigo realizou uma pesquisa de natureza descritiva, com abordagem quantitativa e qualitativa. Em relação aos procedimentos quantitativos, foram empregados instrumentos estatísticos tanto na coleta quanto no tratamento dos dados. Foram selecionados periódicos pertencentes aos estratos B1 e B2 da base Qualis/Capes, visando a identificar tendências em relação aos procedimentos teórico-metodológicos, baseados no modelo proposto por Burrel e Morgan (1979), concentrando-se em publicações nacionais sobre o tema da estratégia organizacional. A abordagem qualitativa, por sua vez, complementou as análises quantitativas, por meio do aprofundamento da compreensão do fenômeno investigado, detendo-se em aprofundar aspectos não observados nos resultados anteriores, assim como realizando interpretações e inferências subjetivas. A análise dos dados empíricos (dados secundários) seguiu o método proposto por Miles e Huberman (1994), que consiste na condensação e na apresentação dos dados na forma de quadros e tabelas.

Em relação ao instrumento de pesquisa, utilizou-se a análise documental, com intuito de agrupar informações extraídas dos artigos publicados nos periódicos selecionados. Esse tipo de abordagem aliada à pergunta de pesquisa, que orienta o presente estudo, R. eletr. estrat. neg., Florianópolis, v.5, n.3, p. 259-279, set./dez. 2012 
possibilitou uma leitura enriquecida das informações obtidas. Por fim, este estudo alinha-se com o paradigma interpretativo de pesquisa, cuja abordagem epistemológica pressupõe a inter-relação do investigador com o seu objeto de pesquisa, na medida em que o sujeito do conhecimento compartilha e integra o contexto estudado (BURREL; MORGAN, 1979).

Haja vista que o objetivo do presente estudo pretendeu avaliar artigos com base em indicadores bibliométricos, foram necessárias diferentes ações para o seu delineamento. Inicialmente, definiu-se que o objeto da presente pesquisa concentra-se em investigar as publicações nacionais consideradas como o "estado da arte" sobre temas relacionados à estratégia organizacional. Na busca deste objetivo, foram selecionados periódicos nacionais dos estratos B1 e B2 na área de Administração, Ciências Contábeis e Turismo, conforme classificação estabelecida pela Capes. Posteriormente, foram selecionadas as seguintes palavras-chave associadas ao tema: "administração" e "gestão". Com a definição dos periódicos, procedeu-se a fase seguinte do estudo. Definiu-se a palavra-chave "estratégia" e partiu-se para a seleção dos artigos por meio de busca nas publicações selecionadas, inserindo-se no comando "pesquisar" dos sites dos periódicos o termo "estratégia". Após esta etapa, foram definidos critérios para orientar a análise. Decidiu-se selecionar artigos que tratassem da estratégia organizacional como tema central, e que contivessem explicitamente, no título do artigo, a palavra "estratégia". Os periódicos cujo acesso à sua base de artigos não fosse livre, ou seja, periódicos pagos foram desconsiderados. Destaca-se que a seleção dos artigos foi realizada após a divulgação da nova classificação dos periódicos científicos por estratos, neste caso específico, B1 e B2 classificados pela Capes em 2012.

A partir dos critérios adotados, chegou-se a um resultado de 543 artigos no estrato B1 e 117 no estrato B2, perfazendo um total de 660 artigos. Após a leitura dos títulos e resumos de cada artigo, foram encontrados 86 artigos nos periódicos selecionados que tratavam do tema "estratégia organizacional". Uma síntese do desenvolvimento da pesquisa é apresentada na Figura 2. 
Figura 2 - Design da Pesquisa

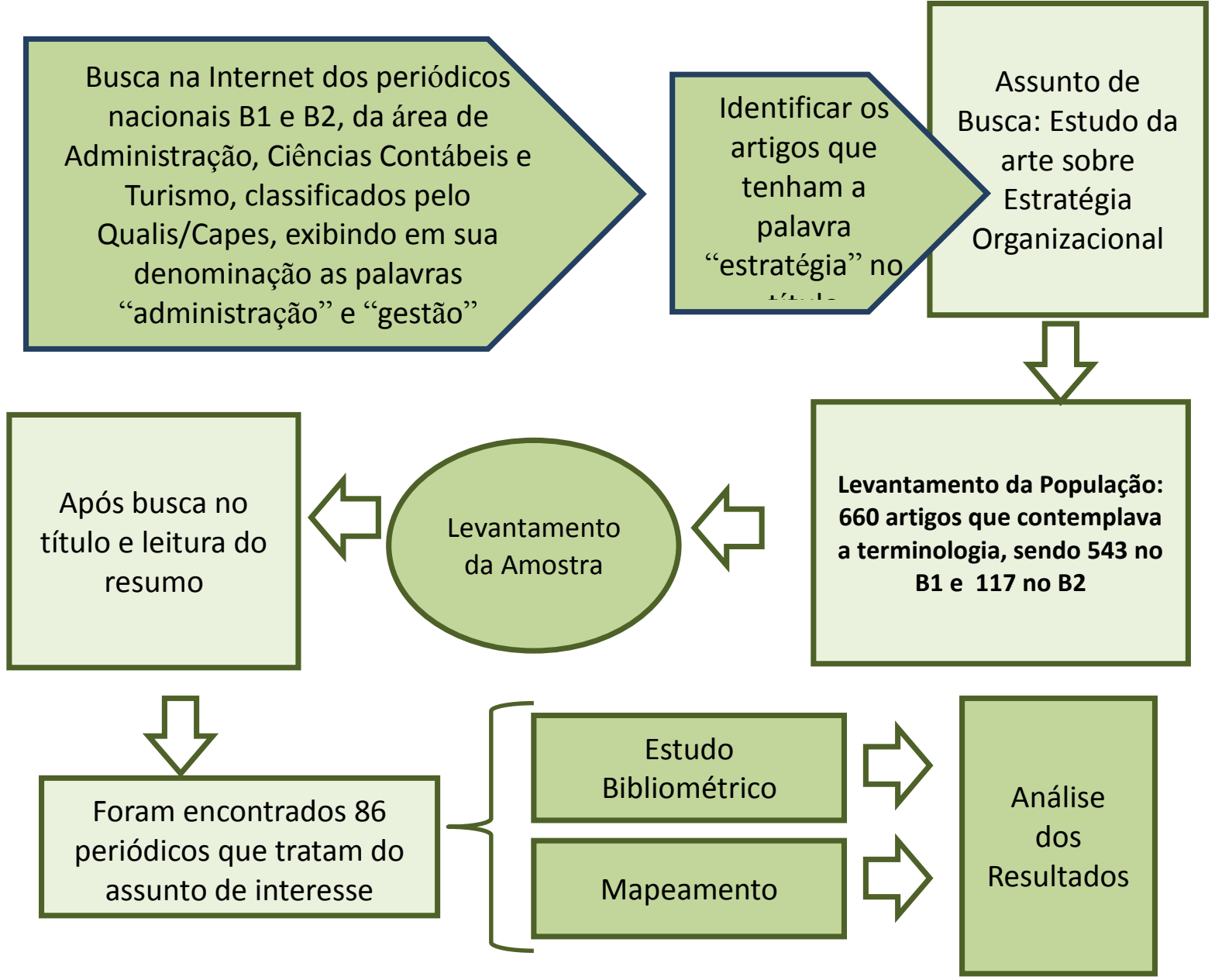

Fonte: Adaptado de Nascimento, Bortoluzzi e Dutra (2009, p. 6)

De posse dos artigos selecionados, os dados foram tabulados e fez-se a análise dos resultados obtidos, que será apresentada na seção 4.

\section{ANÁLISE BIBLIOMÉTRICA DOS DADOS}

Inicialmente, é apresentado um panorama geral dos artigos identificados por este estudo (Tabela 1). Na pesquisa realizada, foi encontrado um total de 86 artigos que contemplavam a palavra-chave "estratégia", conforme os procedimentos descritos na seção 3. 
Tabela 1 - Artigos Identificados

\begin{tabular}{c|c|c}
\hline ESTRATO & ARTIGOS ENCONTRADOS & (\%) \\
\hline B1 & 543 & 82,27 \\
\hline B2 & 117 & 17,73 \\
\hline TOTAL & $\mathbf{6 6 0}$ & $\mathbf{1 0 0 \%}$ \\
\hline
\end{tabular}

Fonte: Elaborado pelos autores, 2012.

A partir dos artigos identificados, foram eliminados, de forma objetiva, os artigos que não possuíam a palavra "estratégia" em seus títulos e, de forma subjetiva, após leitura dos resumos, os artigos que não abordavam o assunto de interesse da pesquisa; ou seja, não tratavam do tema da estratégia organizacional, restando, assim, 86 artigos. Em seguida, apresenta-se na Tabela 2, a amostra analisada, segmentada por estratos.

Tabela 2 - Dados Coletados (Amostras)

\begin{tabular}{c|c|c}
\hline ESTRATO & ARTIGOS & Percentual (\%) \\
\hline B1 & 53 & $62 \%$ \\
\hline B2 & 33 & $38 \%$ \\
\hline TOTAL & $\mathbf{8 6}$ & $\mathbf{1 0 0 \%}$ \\
\hline
\end{tabular}

Fonte: Elaborado pelos autores, 2012.

As Tabelas 1 e 2 revelam que dos 660 artigos identificados, apenas 86 estão de acordo com os critérios estabelecidos nesta pesquisa. Com base na Tabela 2, verifica-se que, do total de artigos analisados, $62 \%$ correspondem aos periódicos classificados no estrato B1. Diante da amostra coletada, composta por 86 artigos, algumas variáveis analisadas merecem destaque: (i) periódicos com maior publicação sobre o tema; (ii) número de publicações por ano; (iii) temas dos artigos; (iv) autores mais citados nos artigos; (v) paradigma dominante; (vi) teoria que fundamenta o artigo; e, (vii) terminologias características do paradigma.

Na Tabela 3, apresentam-se os periódicos com maior publicação sobre o tema da pesquisa. Observa-se uma maior publicação de artigos sobre o assunto estudado no periódico da RAUSP, com 19\% dos artigos selecionados, seguidos pela Revista Gestão \& Planejamento, e Revista Brasileira de Gestão e Negócio, com 15\% cada uma. 
Tabela 3 - Periódicos com maior publicação sobre o tema

\begin{tabular}{|c|c|c|}
\hline PERIÓDICO & QUANT. & $\%$ \\
\hline RAUSP & 16 & $19 \%$ \\
\hline Gestão \& Planejamento & 13 & $15 \%$ \\
\hline Revista Brasileira de Gestão e Negócio - RBGN & 13 & $15 \%$ \\
\hline Revista de Administração Mackenzie - RAM & 8 & $9,5 \%$ \\
\hline Revista Economia \& Gestão - E\&G & 7 & $8 \%$ \\
\hline Revista Portuguesa e Brasileira de Gestão & 7 & $8 \%$ \\
\hline Revista Administração em Diálogo & 6 & $7 \%$ \\
\hline Revista de Administração e Inovação - RAI & 4 & $4,5 \%$ \\
\hline Revista Gestão Organizacional & 4 & $4,5 \%$ \\
\hline Revista Brasileira de Gestão e Desenvolvimento Regional - RBGDR & 3 & $3,5 \%$ \\
\hline Revista de Ciências da Administração - RCA/UFSC & 1 & $1 \%$ \\
\hline Revista de Administração Contemporânea - RAC & 1 & $1 \%$ \\
\hline Revista de Gestão da Tecnologia e Sistemas de Informação & 1 & $1 \%$ \\
\hline Revista de Gestão Social e Ambiental & 1 & $1 \%$ \\
\hline Revista Contabilidade, Gestão e Governança & 1 & $1 \%$ \\
\hline TOTAL & 86 & $100 \%$ \\
\hline
\end{tabular}

Fonte: Elaborado pelos autores (valores arredondados), 2012.

Observando a quantidade de artigos publicados por ano dentre os que abordam o tema da estratégia organizacional, verifica-se que ao longo do período investigado, como mostrado na Tabela 4, há uma tendência crescente de publicações relacionadas ao tema proposto.

Tabela 4 - Número de publicações por ano

\begin{tabular}{r|c|r}
\hline ANO & ARTIGOS & \multicolumn{1}{c}{$\%$} \\
\hline 2012 & 7 & $8 \%$ \\
\hline 2011 & 16 & $19 \%$ \\
\hline 2010 & 11 & $7 \%$ \\
\hline 2009 & 6 & $10 \%$ \\
\hline 2008 & 9 & $13 \%$ \\
\hline 2007 & 11 & $6 \%$ \\
\hline 2006 & 5 & $8 \%$ \\
\hline 2005 & 7 & $7 \%$ \\
\hline 2004 & 6 & (continua)
\end{tabular}


Tabela 4 - Número de publicações por ano

\begin{tabular}{|c|c|c|}
\hline ANO & ARTIGOS & $\%$ \\
\hline 2003 & 3 & $3,5 \%$ \\
\hline 2002 & 3 & $3,5 \%$ \\
\hline 2001 & 2 & $2 \%$ \\
\hline TOTAL & 86 & $100 \%$ \\
\hline
\end{tabular}

Fonte: Elaborado pelos autores (valores arredondados), 2012.

Um interessante aspecto verificado diz respeito aos paradigmas de pesquisa aos quais os artigos estão filiados. Destaca-se o paradigma funcionalista com $78 \%$ dos artigos selecionados, seguido pelo paradigma interpretativista com $15 \%$. Observa-se que não foram encontrados artigos que possuíssem indícios ou evidências que os caracterizassem como filiados aos paradigmas estruturalista radical ou humanista radical, que também compõem o modelo proposto por Burrel e Morgan (1979). Infere-se, então, conforme apontam os resultados da Tabela 5, que a maioria dos artigos pesquisados encontra-se no quadrante da objetividade e da sociologia da regulação.

Tabela 5 - Paradigmas Dominantes

\begin{tabular}{c|c|c}
\hline PARADIGMA & ARTIGOS ENCONTRADOS & $\mathbf{\%}$ \\
\hline Funcionalista & 67 & $\mathbf{1 5}$ \\
\hline Interpretativista & 13 & 7 \\
\hline Sem classificação & 6 & $\mathbf{1 0 0 \%}$ \\
\hline TOTAL & $\mathbf{8 6}$ & \\
\hline
\end{tabular}

Fonte: Elaborado pelos autores, 2012.

O mapeamento possibilitou, ainda, conhecer os autores mais citados nas referências bibliográficas, com intuito de evidenciar aqueles mais influentes no que se refere à estratégia organizacional e que servem como base para as pesquisas nesta área. No total dos 67 artigos classificados no quadrante funcionalista, verificou-se que Michael Porter é o mais citado. Conclui-se que o autor exerce grande influência nos estudos sobre o assunto. Este resultado vai ao encontro da pesquisa realizada por Carneiro et al. (2009) que constatou que nos estudos sobre estratégia organizacional é recorrente a utilização de obras de Porter, pois servem como modelos sobre este assunto, mesmo trinta anos após o lançamento de 
seu artigo seminal. Na sequência, destacam-se, também, os autores Barney, Kaplan e Norton, e Mintzberg.

Tabela 6 - Autores mais citados nos artigos funcionalistas

\begin{tabular}{|c|c|}
\hline AUTORES MAIS CITADOS & № DE CITAÇÕES \\
\hline PORTER, Michael. & 50 \\
\hline BARNEY, Jay. & 19 \\
\hline KAPLAN, Robert S.; NORTON, David P. & 19 \\
\hline MINTZBERG, Henry. & 18 \\
\hline HAMEL, Gary; PRAHALAD, C.K. & 16 \\
\hline MINTZBERG, H. ; AHLSTRAND, B. e LAMPEL, J. & 13 \\
\hline ANSOFF, H. I. & 11 \\
\hline SCHUMPETER, T.S.J.A. & 11 \\
\hline WHITTINGTON, Richard. & 10 \\
\hline HITT, M.A.; IRELAND, R.D.; HOSKISSON, R.E. & 9 \\
\hline PRAHALAD, C.K.; HAMEL, G. & 9 \\
\hline DRUCKER, P, F. & 8 \\
\hline MINTZBERG, H.; QUINN, J. B. & 8 \\
\hline WERNERFELT, Birger. & 8 \\
\hline CHANDLER. A. D. Jr. & 7 \\
\hline RUMELT, R. P. & 7 \\
\hline SIMON, H.A. & 7 \\
\hline GRANT, R. M. T & 6 \\
\hline HALL, Richard. T & 6 \\
\hline MILES, R.; SNOW, C. & 6 \\
\hline PETTIGREW, A. M. & 6 \\
\hline
\end{tabular}

Fonte: Elaborado pelos autores, 2012.

A mesma análise foi realizada em relação aos 13 artigos pertencentes ao quadrante interpretativista. Destacam-se os autores Richard Whittington, sendo citado em 8 dos artigos analisados, seguido por Paula Jarzabkowski (7 artigos); Michael Porter e Mintzberg (6 artigos). Verifica-se que Richard Whittington e Paula Jarzabkowski são autores que possuem como tema de pesquisa a estratégia como prática, que, neste sentido, pode ser considerada como filiada ao paradigma interpretativista. Segundo Pagagnan, Gimenez e Rubo (2012) a estratégia como prática, por guardar em sua essência as particularidades e os eixos basilares da realidade social, aponta caminhos e alternativas concretas para enquadrar 
este tipo de abordagem no paradigma interpretativista. Segue abaixo a lista dos autores citados nos artigos selecionados no paradigma interpretativista.

Tabela 7 - Autores mais citados nos artigos interpretativistas

\begin{tabular}{l|c}
\hline \multicolumn{1}{c|}{ AUTORES MAIS CITADOS } & № DE CITAÇÕES \\
\hline WHITTINGTON, R.. & 8 \\
\hline JARZABKOWSKI, P. & 7 \\
\hline PORTER, Michael. & 6 \\
\hline MINTZBERG. H. & 6 \\
\hline JOHNSON, G.; MELIN, L.; WHITTINGTON, R. & 5 \\
\hline JOHNSON, G.; LANGLEY, A.; MELIN, L.; WHITTINGTON, R & 5 \\
\hline CHIA, R. & 4 \\
\hline PETTIGREW, A. & 4 \\
\hline CLEGG, S.; CARTER, C.; KORNBERGER, M. A & 3 \\
\hline
\end{tabular}

Fonte: Elaborado pelos autores, 2012.

Em relação aos temas mais abordados, verifica-se na Tabela 8 que dentre os artigos selecionados, 18 abordam o tema da estratégia competitiva de diferenciação, sendo seguido pelo tema da estratégia enquanto prática (7 artigos). Novamente constata-se a presença marcante de Michel Porter como autor de destaque sobre o tema da estratégia competitiva. A análise identificou, ainda, a presença do tema sobre a estratégia competitiva de custo, em dois dos artigos selecionados.

Tabela 8 - Temas dos artigos

\begin{tabular}{l|c}
\multicolumn{1}{c|}{ TEMA } & QUANT. \\
\hline Estratégia competitiva de diferenciação & 18 \\
\hline Estratégia enquanto prática & 6 \\
\hline Alianças estratégicas & 4 \\
\hline Estratégia de cooperação & 4 \\
\hline Estratégias nas micro e pequenas organizações & 4 \\
\hline Planejamento estratégico formal & 3 \\
\hline Responsabilidade social estratégica & 3 \\
\hline Estratégias voltadas à sustentabilidade & 2 \\
\hline Estratégia competitiva de custo & 2 \\
\hline Balanced scorecard & 2 \\
\hline Gestão de processos & 2 \\
\hline Fusões e aquisições & 29 \\
\hline Outras abordagens & 86 \\
\hline TOTAL & \\
\hline Fonte: Elaborado pelos autores, 2012. & \\
\hline
\end{tabular}

Fonte: Elaborado pelos autores, 2012. 
Na sequência, nota-se que os autores dos artigos pesquisados não evidenciam com clareza a teoria que visa a suportar as análises empíricas de seus estudos (Tabela 9). A grande maioria dos artigos não explicita uma teoria, nem tão pouco evidencia as características que possam indicar a linha teórica adotada. Esse número ínfimo de artigos que se vale de teorias para fundamentar as suas respectivas abordagens, revela a debilidade teórica e conceitual dos estudos científicos nesta área.

Tabela 9 - Teorias Explicitadas

\begin{tabular}{l|c}
\multicolumn{1}{c|}{ TEORIA } & ARTIGOS ENCONTRADOS \\
\hline Teoria da Complexidade & 2 \\
\hline Teoria Baseada em Recursos & 2 \\
\hline Teoria Institucional & 1 \\
\hline Teoria dos Recursos e da Capacidade & 1 \\
\hline Teoria das Representações Sociais & 1 \\
\hline Teoria das Ações Coletivas & 1 \\
\hline Teoria dos Jogos & 1 \\
\hline Teoria da Estruturação & 1 \\
\hline Teoria da Ação Humana Eficiente & 1 \\
\hline Teoria da Gestão Estratégica & \multicolumn{1}{c}{ TOTAL } \\
\hline
\end{tabular}

Fonte: Elaborado pelos autores, 2012.

Por último, procurou-se identificar indícios, através de palavras-chave e termos, que pudessem caracterizar a filiação dos artigos ao paradigma funcionalista e interpretativista. Após a identificação desses dois paradigmas principais, por meio da leitura integral dos artigos, foram selecionadas palavras, que revelassem alinhamento com os pressupostos teórico-metodológicos de cada um deles (Tabela 5). Encontrou-se, então, 45 termos e/ou palavras após a análise dos 67 artigos funcionalistas selecionados. De posse dessas palavras, procurou-se verificar a presença de cada um desses 45 termos e/ou palavras nos 67 artigos funcionalistas. Em seguida, foram selecionados aqueles termos e/ou palavras que estavam presentes em, pelo menos, três artigos. Foram encontrados 15 termos e/ou palavras que atendessem a este critério. Fez-se uma nova seleção, desta vez utilizando a ferramenta "localizador" do Word para encontrar nos 67 artigos funcionalistas, as 15 palavras selecionadas, cujo resultado está indicado na Tabela 10. 
Tabela 10 - Resultado dos termos e/ou palavras selecionadas nos 67 artigos funcionalistas

\begin{tabular}{l|c|c}
\multicolumn{1}{c|}{ Palavras selecionadas } & Frequência & \% \\
\hline Tecnologia & 64 & 96 \\
\hline Mercado & 61 & 91 \\
\hline Estrutura & 58 & 87 \\
\hline Recursos & 56 & 84 \\
\hline Vantagem competitiva & 48 & 72 \\
\hline Competitividade & 47 & 70 \\
\hline Inovação & 45 & 67 \\
\hline Capital & 37 & 55 \\
\hline Desenvolvimento de produto & 25 & 37 \\
\hline Planejamento estratégico & 23 & 34 \\
\hline Estratégia competitiva & 19 & 28 \\
\hline Posicionamento estratégico & 11 & 16 \\
\hline Estratégia de diferenciação & 11 & 16 \\
\hline Desempenho econômico & 5 & 7 \\
\hline Mercado competitivo & 4 & 6 \\
\hline
\end{tabular}

Fonte: Elaborado pelos autores (valores arredondados), 2012.

Analisando a Tabela 10, vê-se que os termos "competitividade" (70\%) e "mercado" (91\%), dentre outros, são evidências que reforçam o posicionamento dos artigos dentro do paradigma funcionalista, caracterizado por abordagens objetivistas que visam ao aperfeiçoamento dos processos organizacionais, ou seja, que buscam a regulação. $\mathrm{O}$ aparecimento do termo "vantagem competitiva" em 71\% dos artigos funcionalistas revela a tendência nas últimas três décadas da presença de trabalhos influenciados pelos conceitos de Michel Porter, que é o autor mais citado nestes artigos. Nesse sentido, reforça-se a percepção de que os estudos da área de estratégia organizacional recebem grande influência de abordagens economicistas como as de Michael Porter.

Em relação aos termos "tecnologia" (presente em 96\% dos artigos) e "inovação" (67\%), pode-se afirmar a existência de um vínculo profundo entre estes processos (tecnologia e inovação) com os atributos que concorrem para a conquista da vantagem competitiva das organizações. Ou seja, as abordagens funcionalistas, em geral, consideram que a tecnologia e a inovação estão vinculadas à vantagem competitiva. Isto revela uma visão profundamente reducionista das abordagens dominantes, pois tanto a inovação quanto a tecnologia representam um conjunto de processos muito mais amplos e diversos, R. eletr. estrat. neg., Florianópolis, v.5, n.3, p. 259-279, set./dez. 2012 
que afetam não somente as organizações, que visam construir vantagens competitivas no mercado concorrencial, mas, também, outras, tais como os institutos públicos de pesquisa, cujos objetivos transcendem a conquista da vantagem competitiva.

A mesma análise foi realizada nos 13 artigos enquadrados no paradigma interpretativista. Após a leitura integral dos textos, foram escolhidos 37 termos. Utilizando a mesma técnica, foram selecionados 9 termos que estavam presentes em, pelo menos, três artigos. Igualmente, repetiu-se o processo de busca através da ferramenta "localizador" do Word, cujo resultado é apresentado na Tabela 11.

Tabela 11 - Resultado dos termos e/ou palavras selecionadas nos 13 artigos interpretativistas

\begin{tabular}{l|c|c}
\hline Palavras selecionadas & Frequência & $\%$ \\
\hline Atores & 12 & 92 \\
\hline Pessoas & 11 & 85 \\
\hline Indivíduos & 10 & 77 \\
\hline Agentes & 8 & 62 \\
\hline Práticas sociais & 7 & 54 \\
\hline Estratégia como prática & 6 & 46 \\
\hline Teoria social & 4 & 31 \\
\hline Estrutura social & 3 & 23 \\
\hline
\end{tabular}

Fonte: Elaborado pelos autores (valores arredondados), 2012.

Conforme pode ser observado, o paradigma interpretativista está intimamente vinculado a atributos e componentes que caracterizam o mundo social, evidenciados pela presença de termos, tais como "pessoas", "indivíduos", "práticas sociais" etc. Este resultado confirma a relação deste paradigma com os procedimentos dialógicos que vinculam a relação entre sujeito e objeto do conhecimento.

\section{CONSIDERAÇÕES FINAIS}

O levantamento quantitativo-qualitativo realizado por meio de um estudo bibliométrico resultou no mapeamento de informações relevantes sobre o tema da estratégia organizacional, apresentadas ao longo deste trabalho. Identificou-se nos 
periódicos selecionados que os autores mais citados nos artigos e que mais têm contribuído nesta área de pesquisa são Michael Porter, Michael Mintzberg e Richard Whittington. Em relação à linha teórica seguida, não houve destaque, tendo em vista o pequeno número de artigos que explicitam as suas fundações teóricas.

A relevância do estudo aqui desenvolvido está na contribuição dos resultados encontrados, que buscou oferecer à área de administração um mapeamento das publicações, concentradas nos estratos B1 e B2 do sistema Qualis/Capes, bem como, fornecer informações acerca dos paradigmas sociológicos utilizados nos estudos que abordam a estratégia organizacional. A predominância do paradigma funcionalista foi evidenciada neste estudo, como já havia sido confirmada em outros trabalhos anteriores (CALDAS; BARTERO, 2007; CRUZ; PEDROZO, 2008).

Neste sentido, acredita-se que a presente pesquisa poderá orientar novos estudos com vistas a uma eventual expansão do mapeamento aqui apresentado, por meio de pesquisas em outros estratos do sistema Qualis/Capes, assim como em outras bases de dados, tais como Cielo e Ebsco.

\section{MAPPING OF EPISTEMOLOGICAL APPROACHES ON PUBLICATIONS ON THE ORGANIZATIONAL STRATEGY FIELD}

\section{ABSTRACT}

This paper aims to conduct a study about the major epistemological approaches, identified in articles on the subject of organizational strategy. The theoretical framework that guided this research is based on the classical definition proposed by Burrell and Morgan (1979) about the four paradigms of social science research. We investigated articles in the area of Administration, Accounting and Tourism, published in Brazilian journals between the years 2001 to 2012, classified as "B1" and "B2" in accordance with Qualis / CAPES criteria. In order to meet this goal, we performed a bibliometric study to map the selected publications. As a result of this study, there was a predominance of the functionalist paradigm, as well as a reduced presence of articles which explain their theoretical and methodological choices as the foundation for their empirical studies. 
MAPEAMENTO DE ABORDAGENS EPISTEMOLÓGICAS EM PUBLICAÇÕES SOBRE ESTRATÉGIA ORGANIZACIONAL

Renê Birochi - Maria Zenilda da Silva - Flavia Domingues - Amilton Bento - Richeli Sacheti

Keywords: Organizational Strategy; Paradigms in the Social Science; Theory of Organizations; Epistemology.

\section{REFERÊNCIAS}

BOURDIEU, P. Understanding. Theory, Culture, and Society, v. 2, n. 13, 1996, p. 17-36.

BURREL, G.; MORGAN, G. Sociological Paradigms and Organizacional Analysis. Heineman: London, 1979.

CALDAS, Miguel P.; BERTERO, Carlos Osmar (org.) Dados Internacionais de Catalogação na Publicação (CIP). (Câmara Brasileira do Livro, SP, Brasil) - São Paulo: Atlas, 2007.

CALDAS, P. Miguel. Paradigmas em Estudos Organizacionais: Uma Introdução à Série. RAE, vol. 45, número 1, 2005.

CARNEIRO, M. L.; SCHNEIDER, A.B.; SERRA, F. R.; FERREIRA, M. P. A Influência de Michael Porter Na Estratégia Brasileira 30 Anos Depois de Estratégia Competitiva. Rev. Adm. UFSM, Santa Maria, v. 2, n. 2, 2009.

CHAUÍ, Marilena. Convite à Filosofia. São Paulo: Ática, 2000.

CRUZ, L. B; PEDROZO, E. A. Pesquisas de concepção como uma alternativa para o campo da estratégia. Revista de Administração Mackenzie -- RAM, v. 9, n. 4, edição especial, 2008, p. 56-74.

KUHN, T. S. The structure of scientific revolutions. Chicago: University of Chicago Press, 1962.

MASTERMAN, M.: The Nature of a Paradigm. In I. Lakatos \& A. Musgrave (eds.), Criticism and the Growth of Knowledge, Cambridge University Press, Cambridge, UK, pp. 59-89, 1970

MEIRELLES, Anthero de Moraes. O que é estratégia: uma abordagem multiparadigmática para a Disciplina. Editado pelo Departamento de Estudos e Pesquisas (DEPEP), 2005.

MILES, M.; HUBERMAN, A. Qualitative data analysis: an expanded sourcebook. Thousand Oaks: Sage Publications, 1994. 
NASCIMENTO, S.; BORTOLUZZI, S.C.; DUTRA, A. Mapeamento dos Indicadores de Desempenho Organizacional em Pesquisas da área de Administração, Ciências Contábeis e Turismo, no período de 2000 a 2008. Encontro da ANPAD. São Paulo-SP 2009.

PACAGNAN, M. N; GIMENEZ, F. A.; RUBO, M. Estratégia como Prática: uma alternativa de pesquisa a partir do paradigma interpretativista e suas cataegorias. ADM v. 5, n.1, 2012.

PERRET. V; SEVILLE, M. Fondements épistémologiques de la recherche. In: THIETART, R. A. et al (Org). Méthodes de rechercheemmanagement. Paris: Dunod, 2003, p. 13-33.

PINTO, M. de R.; SANTOS, L. L. da S. Em busca de uma trilha interpretativista para a pesquisa do consumidor: uma proposta baseada na fenomenologia, etnografia e na grounded theory. RAE Eletrônica, v. 7, n. 2, 2008.

PRASAD, P. Crafting qualitative research: working in the postpositivist traditions. Armonk NY: M.E. Sharpe, 2005.

RICHARDSON, Roberto Jarry. Pesquisa social: métodos e técnicas. 3. ed. São Paulo: Atlas, 2008.

SERVA, M.; DIAS T.; ALPERSTEDT, G. D.. Paradigma da complexidade e teoria das organizações: uma reflexão epistemológica. RAE Eletrônica, v. 50, n. 3, 2010.

THIOLLENT, M. Metodologia da pesquisa-ação. São Paulo: Cortez, 1996. 